papers were discussed at plenary sessions and at meetings of all the commissions held during the period of the Conference.

The Institute publishes six times a year a Bulletin which appears in both English and French, the two official languages of the Institute. The Bulletin contains abstracts of scientific and technical articles and information about current research in refrigeration and on other refrigeration activities from all over the world. The Institute also publishes the works of its various commissions.

Full membership of the Institute is restricted to the governments of member countries, which at present number 35, including the United Kingdom, the United States and the U.S.S.R. The United Kingdom interests in the Institute are co-ordinated by the Department of Scientific and Industrial Research, with the advice of a Standing Committee representing research, institutional and industrial interests in Great Britain.

In 1952, the Institute introduced associate membership, available to qualified firms, institutions or individuals active in the science or in the industry of refrigeration. The annual subscription is about $£ 13$ for firms and institutions and $£ 310 s$. for individuals. Associate members receive the Bulletin and the proceedings of the nine international com. missions of the Institute, together with the texts of reports presented. Associate members may participate in the work and the meetings of the commissions in which they are interested, and can also use the services of the large library of the Institute. Applications for associate membership may be made to the Director of the International Institute of Refrigeration, 177 boulevard Malesherbes, Paris $\left(17^{\circ}\right)$.

\title{
STERIC ASPECTS OF THE CHEMISTRY AND BIOCHEMISTRY OF NATURAL PRODUCTS
}

$\mathrm{T}$ HE interest taken in stereochemical problems by chemists and biochemists alike has been greatly increased in recent years, and the Biochemical Society recognized this fact by arranging a symposium on "Steric Aspects of the Chemistry and Biochemistry of Natural Products", which was held in the Senate House of the University of London on June 30. The chairman of the morning session, Prof. A. Neuberger (London), discussed some of the main trends of recent work in this field and emphasized the importance of stereochemistry in modern enzymology.

Dr. W. Klyne (London) then discussed in a comprehensive manner the types of evidence used for establishing relative or absolute configurations of asymmetric compounds. The term 'absolute configuration' can now be used with confidence, as Bijvoet and his colleagues working in Utrecht have demonstrated by means of a special X-ray technique that the Fischer convention for glyceraldehyde happens to be correct. Dr. Klyne pointed out that the most satisfactory method of correlating two asymmetric compounds is by a chemical reaction which does not involve the asymmetric centre. The second type of approach is concerned with chemical reactions in which one or more of the linkages of the asymmetric atoms are broken. The stereochemical correlation in this situation must be based on kinetic and other evidence and must involve certain assumptions about the mechanisms of the substitution concerned.

Dr. Klyne then went on to discuss the deductions which can be made from studies of asymmetric synthesis, methods used successfully by Prelog and by Cram. Another type of approach which was developed mainly by Fredga in Sweden, and which is probably not sufficiently widely known, is based on the study of melting points of mixtures of a compound of known configuration and a structurally similar compound of unknown configuration. If the two compounds have opposite configurations they may form in the solid phase a quasi-racemic compound, and this can be deduced from the melting-point curve.

Reference was also made to the information obtained from the applications of X-ray analysis, especially to compounds with more than one asymmetric centre. Finally methods were discussed which depend on a numerical comparison of the values of optical rotations of structurally related compounds. These calculations and deductions have in the past been largely based on measurements at a single wave-length and have indeed yielded much valuable information. In recent years this tool has been made more powerful by extending the measurements to the whole visible and a large part of the ultra-violet range of the spectrum. In this development (rotatory dispersion), Djerassi of Detroit has taken the leading part.

Steric aspects of the biosynthesis of terpenes and steroids were considered by Dr. D. Arigoni (Zurich), who discussed first the formation of an isopentane derivative from acetate. The early stages of the synthesis consist of a condensation of acetoacetyl-CoA with acetyl-CoA to give the CoA derivative of $\beta$-hydroxy- $\beta$-methylglutaric acid. The latter is then reduced, probably through the aldehydo acid, to $\beta, \delta$-dihydroxy- $\beta$-methylvaleric acid or movalonic acid. The absolute configuration of this compound has been unambiguously related recently by Eberle and Arigoni to that of quinic acid, which in turn had been established by Dangschat and Fischer in 1950 by relating it to glyceraldehyde. Dr. Arigoni then referred to the stereochemical problems involved in the conversion of leucine to $\beta$-hydroxy- $\beta$-methylglutaconic acid, which occurs through the CoA esters of $\beta$-mothylcrotonic acid and $\beta$-methylglutaconic acid. The hydration of the double bond and the carboxyla. tion of methylcrotonic acid must be stereospecific and this is also likely to apply to the reduction of mevaldic acid to mevalonic acid. The next steps in the reaction sequence are the simultaneous decarboxylation and elimination of the tertiary hydroxyl group from the pyrophosphate of mevalonic acid to give isopentenyl pyrophosphate and the isomerization of the latter to give dimethylallyl pyrophosphate; it can be postulated that the isomerization is stereospecific and Dr. Arigoni thought it probable that only one of the two hydrogen atoms of iso. pentenyl pyrophosphate is involved in the isomerization. 
The allyl compound is assumed to react with the isopentenyl pyrophosphate, resulting in the formation of $a$ new $\mathrm{C}-\mathrm{C}$ bond. This reaction must again have stereospecificity, since the new double bond produced usually has a trans configuration; but Dr. Arigoni stressed the fact that the detailed mechanism of the formation of this condensation is not yet securely established and he suggested that experiments involving labelling with deuterium and determining the axial or equatorial position of the deuterium in a suitable cyclization product are likely to give further information.

Dr. Arigoni pointed out that steric factors had to be taken into account in any attempts which were made to explain the actual cyclization and the rearrangements which either follow the cyclization proper or are coupled with it. Otherwise, it would be difficult to explain the formation of diastereoisomers from one single aliphatic precursor, squalene. If a carbonium ion is involved in cyclization reactions, it can only have a structure which preserves the original configuration; a 'bridged ion' fulfils this requirement and addition of this relatively stable species to a base or nucleophilic substance ('antiplanar addition') will produce only one isomer. The postulate of antiplanar addition imposes restrictions on the type of folding and it appears that only the chair-type and boat-type of folding explain, for example, the formation of both lanosterol and tirucallol from all-trans squalene. Experimental evidence for the theory is provided by the work of Bloch and by that of Cornforth and Popják. Further interesting examples from the work of Dr. Arigoni himself and from that of Prof. Birch were given, showing the application of stereochemical rules to the biogenesis of terpenes, but a note of caution was sounded against the assumption that the configuration of the $A / B$ ring junction is always the same.

Steric aspects of drug action were discussed by Dr. R. B. Barlow (Edinburgh), who began his talk by emphasizing the distinction first made by Stephenson between affinity or adsorbability of a drug to a receptor site, and efficacy, that is, the ability of the adsorbed drug to start a sequence of reactions which can be observed in a pharmacological experiment. While it is possible to make definite statements about the steric arrangement of drugs, ideas on the stereochemistry of receptors are generally based on somewhat uncertain deductions made from structures of active compounds and those of their antagonists.

Dr. Barlow illustrated his talk with examples from the field of drugs resembling acetylcholine either in its muscarine-like or nicotine-like function or of compounds antagonizing such action. For nicotinelike activity the molecule should contain a cationic head such as a dimethylemino or trimethylamino group and a partial positive charge at a distance similar to that which separates the ether oxygen of acetylcholine from the charged nitrogen atom. In nicotine itself the two optical isomers have identical pharmacological activities. The constitution and stereochemistry of muscarine have recently been worked out and it is found that it has three centres of asymmetry. Dr. Barlow then discussed the activities of the various stereo-isomers of muscarino and of various synthetic substrates resembling this substance pharmacologically, such as acetyl- $\beta$-methylcholine. In this case the $(+)$-isomer is reported to be about 200 times as active as the $(-)$-isomer. Dr. Barlow then mentioned the importance of steric factors in flexible molecules and he pointed out that in these cases it is more difficult to arrive at quantitative conclusions. In particular, the work of Sehueler was discussed in detail and some recent criticisms of his calculations were mentioned. Dr. Barlow then dealt in some detail with di-quaternary bases, such as various esters of aliphatic dicarboxylic esters containing two quaternary basic groups, such as suxamethonium. The many examples which were considered illustrated the difficulties which still exist if one attempts to correlate the stereochemistry of the compounds under consideration with their biological activity.

The last paper of the morning session was given by Dr. G. A. J. Pitt (Liverpool) on behalf of Prof. R. A. Morton and himself, and dealt with cis-trans isomers of retinene in visual processes. Dr. Pitt briefly discussed the chemistry of retinene (vitamin A aldehyde) and that of vitamin $\mathbf{A}$, and in particular referred to the early work of Pauling which predicted the existence of the following four isomers: alltrans; 9-monocis; 13-monocis; and 9:13-dicis. These four isomers of retinene and vitamin $A$ have been synthesized, but it has been possible to prepare two other retinenes and vitamins $A$ containing a cis-linkage in the 'hindered' 11-position. No 7-cis vitamin A has yet been prepared and it seems almost certain that 7-cis isomers cannot exist, as in such a molecule there would be considerable steric interference. Dr. Pitt then referred briefly to the occurrence of eis-isomers in Nature, and mentioned that the thermodynamically most stable isomer and the one found most commonly in Nature is the all-trans isomer. $\mathrm{H}_{\theta}$ then reviewed the isomerization of the various retinene isomers and their absorption spectra. The importance of the cis-trans isomerization was appreciated when Hubbard and Wald found that the retinene which united with opsin was the 11-cis form. The 9-cis form also reacted with opsin but the resulting complex has not been found in Nature. The main effect of the introduction of a cis-bond at the 11-position is that it produces a bend of the sidechain whereas in the all-trans isomer the side-chain is straight. When rhodopsin is illuminated it breaks down to the protein opsin and a retinene, but the latter was found, rather surprisingly, to be the alltrans isomer. This all-trans retinene liberated by the bleaching of rhodopsin does not give rhodopsin again when mixed with the opsin in vitro, and strong evidence has been obtained by Hubbard that the utilization of the trans-isomer in a more complex system is caused by the presence of an enzyme called retinene isomerase, which changes the all-trans retinene to the 11-cis isomer. Dr. Pitt discussed this enzymic isomerization in more detail and then went on to report on recent work which has been done on invertebrate rhodopsins, dealing in particular with indicator yellow. He also gave an account of the effect of light on various rhodopsins under a variety of conditions, but it would be impossible to summarize adequately the rather complicated relationships which have been established, mainly due to the work of Hubbard and Wald.

The chair was taken for the afternoon session by Prof. S. Peat (Bangor); all three papers were devoted to various aspects of stereospecificity in enzyme action. Dr. E. C. Webb (Cambridge), who dealt with hydrolytic enzymes, treated some general matters relevant to all enzymes. He emphasized that enzymic catalysis is at least a two-stage process, and that steric factors may be important both in the formation and in the breakdown of the intermediate 
enzyme-substrate complex. To be of real value any comparison of substrates should involve measurement of both the Michaelis constant $\left(K_{m}\right)$ and the reaction velocity, as measures of the two stages.

Examples considered included ali-esterases and lipases, cholinesterases, glycosidases, lactonases, peptidases, arginase and fumarase. For the cholinesterases knowledge has accumulated which permits the representation of the catalysis in terms of anionic and esteratic sites on the enzyme surface. The high specificity of glycosidases is well known, and this is paralleled in the recently studied group of lactonases. Proteolytic enzymes may be used, thanks to their high stereospecificity, as tools for the resolution of synthetic amino-acids. Work on the behaviour of synthetic substrates as competitive inhibitors of $\alpha$-chymotrypsin emphasizes the importance of distinguishing the two stages of enzyme action.

In conclusion, the importance of Ogston's concept (1948) of three-point attachment between enzyme and substrate was emphasized. This idea explains very simply the formation from a symmetrical substrate $\left(\mathrm{C} X_{2} Y Z\right)$ of an asymmetric product $\left(\mathrm{C} X X^{1} Y Z\right)$. Ogston has recently discussed this concept further (1958).

Dr. H. Gutfreund (Shinfield) in discussion considered further the specificity of $\alpha$-chymotrypsin, in the reactions of which three stages can be distinguished $(E+A B \rightleftharpoons E-A B \rightleftharpoons E A+B \rightleftharpoons E+A+B)$.

Prof. E. C. Slater (Amsterdam) dealt with oxidation-reduction enzymes. He began by considering dehydrogenases which act on hydroxy acids and more complex systems which can (indirectly) transform one enantiomer into the other (commonly but wrongly called racemases). After a brief discussion of aminoacid oxidases the greater part of the paper was devoted to stereospecificity of hydrogen transfer in the reactions of pyridine nucleotide dehydrogenases. These compounds are dinucleotides, one unit of which is the nicotinamide group which can undergo reversible oxidation and reduction at $\mathrm{N}$ and $\mathrm{C}-4$. The elegant work of Vennesland's school in Chicago using substrates and enzymes labelled with deuterium has shown that these dehydrogenases fall into two classes ; these are distinguished by the fact that they add hydrogen at $\mathrm{C}-4$ of the diphosphopyridine nucleotide molecule on opposite sides. Other related work dealt with reactions involving eytochrome $c$, flavin and orotic acid.

An important extension of Vennesland's work was the preparation of stereospecifically labelled and optically active $\mathrm{CH}_{3} \cdot \mathrm{CHDOH}$. Alternative mechanisms of action for a typical dehydrogenase have been suggested by Dixon and Webb in their classic textbook on "Enzymes", and by Van Eyk, Kaplan et al.

Finally, succinic dehydrogenase, which involves another type of stereospecificity, was introduced.

Dr. H. R. Levy (Chicago) emphasized in discussion that the stereospecificity in the reactions of pyridine nucleotides is another example of the ability of enzymes to distinguish between two identical groups, and gave further examples. Dr. W. Klyne directed attention to the important work of Prelog on microbiological oxidations and reductions of simple decalones and related compounds. The pictorial treatment of these reactions may constitute a valuable extension of Ogston's concept of three-point attach. ment.

Dr. G. R. Barker (Manchester) dealt with enzymes of nucleotide metabolism. He began by outlining current views on the biosynthesis of D-ribose and 2-deoxy-D-ribose; the important routes leading to ribose involve the 5-phosphates of $\mathrm{D}$-xylulose and D-ribulose. The formation and fission of glycosidic bonds in nucleosides was next considered; these reactions are generally phosphorolyses and not hydrolyses. The stereochemistry of analogous chemical syntheses of nucleosides has been studied with respect to relative configurations at $\mathrm{C}-1$ and $\mathrm{C}-2$, and it is necessary to consider how far the stereo. specificity of the enzymic reaction is attributable to the enzyme, and how far it is inherent in the nature, of the reaction eatalysed. The answer at present is that both factors are involved.

The formation and fission of internucleotide links in polynucleotides were then discussed. Pancreatic ribonuclease degrades ribopolynucleotides via nucleoside $2^{\prime}, 3^{\prime}$-(cyclic)-phosphates to $3^{\prime}$-phosphates, and the steric factors involved in this and the reverse reaction were considered. Finally, the polynucleotide phosphorylases were considered; many reactions require a polynucleotide 'primer', the composition of which determines that of the polymer formed; this is a direct demonstration that the primer acts as a template.

All three papers emphasized in different ways the essentially complementary nature of enzyme and substrate and the two (or more) stage character of the enzyme-substrate reaction.

A. Neuberger W. KrYNE

\section{OIL IN NAVIGABLE WATERS}

T is now seven years since a group of representatives of the interests in Great Britain which are most affected by oil pollution formed themselves into the Co-ordinating Advisory Committee on Oil Pollution of the Sea, under the chairmanship of Mr. James Callaghan, M.P. In 1953 this Committee organized an international conference in London which called for a meeting of governments of all maritime countries to take action to prevent the growing pollution of the oceans of the world. This request met with considerable success, for an inter-governmental conference was held in 1954, at which an international convention was drawn up. This Convention came into force in July 1958, and has been ratified by the
United Kingdom, Belgium, Canada, Denmark, Finland, France, the Federal Republic of Germany, the Irish Republic, Mexico, the Netherlands, Norway and Sweden.

The Convention does not prohibit the discharge of waste oil entirely, but merely within certain zones. and the countries which observe the Convention represent only about one-half of the world's tanker shipping, notable omissions being the United States, U.S.S.R., Poland, Italy, Panama and Liberia. The Co-ordinating Advisory Committee on Oil Pollution of the Sea therefore decided to organize an international conference to discuss, among other aspects, how far the 1954 Convention had been effective in 